\title{
Use of "Lossy" LP-BP-Transformation in Active Second-order BP Filter Design Procedure
}

\author{
Dražen Jurišić and Neven Mijat \\ Faculty of Electrical Engineering and Computing, Unska 3, HR-10000 Zagreb, Croatia. \\ Tel: +385161294 11, Fax: +38516129652 \\ drazen.jurisic@fer.hr;neven.mijat@ffer.hr
}

\begin{abstract}
In this paper the design of second-order band-pass (BP) active $R C$ filters using a modified low-pass to band-pass (LP-BP) frequency transformation is presented. The transformation is applied to a first-order low-pass (LP) filter as the (odd-order-)prototype, from which a single-amplifier second-order BP filter is constructed. The operational amplifier is added to the first-order LP circuit in order to provide a low output impedance and supply a positive feedback loop to enable a pole shifting process needed in the realization. It is shown that a BP filter can be realized by substitution of resistor and capacitor in the low-pass prototype filter, by serial and parallel $R C$ circuits in the resulting bandpass structure. A Schoeffler sensitivity is used as a measure of the magnitude sensitivity to component tolerances. A step-by-step design procedure is verified for several second-order band-pass filter circuits, using different impedance scaling ratios, resulting by different sensitivities. It is shown that the circuit with equal impedance scaling ratios yields the best results. Obtained results are double-checked using PSPICE.
\end{abstract}

\section{Introduction}

The design of BP filters is usually performed by means of the well-known LP-BP frequency transformation applied to a LP prototype filter transfer function [1-4].

In the previous paper [3] we presented a new procedure for the realization of single-amplifier active $R C$ fourth-order BP filter directly from a given secondorder LP prototype structure, using the prototype impedance transformation, which corresponds to the socalled lossy LP-BP transformation [1,2]. Lossy LP-BP transformation is applied to an LP prototype, which has the complex poles shifted to the right-half of the complex frequency plane. The shifting procedure is performed, by increasing the gain $\beta$ of a single amplifier LP prototype.

In this paper we extend the design procedure to the filter, which has a negative real pole in a prototype circuit. In order to shift real pole in the right-half plane by the amount of $\delta$, we made some modifications to the circuit. An operational amplifier is added to the firstorder low-pass (LP) prototype filter circuit, in order to provide positive feedback loop, which enable the pole shifting procedure. The method can be extended to higher-order BP circuits, which have odd-order LP prototypes (e.g. sixth-order BP filter has a third-order LP prototype). Furthermore, it can be shown that by increasing the impedance scaling factors, sensitivity to component tolerances can be significantly reduced [6]. However, describing such impedance scaling goes beyond the scope of this paper.

\section{Design of Second-Order Band-Pass Filters using the LP-BP Transformation}

Consider the first-order passive $R C$ network shown in the Fig. 1.

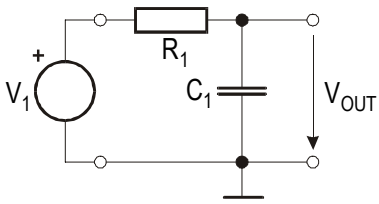

\section{Fig. $11^{\text {st }}$-order passive $R C$ low-pass circuit}

The voltage transfer function $T(s)$ for this circuit is given by

$$
T(s)=\frac{V_{\text {out }}}{V_{1}}=\frac{\left(R_{1} C_{1}\right)^{-1}}{s+\left(R_{1} C_{1}\right)^{-1}}=\frac{\gamma}{s+\gamma}=\frac{K}{\left(s-s_{p 1}\right)}
$$

This function has one negative real pole $s_{p 1}=-\gamma$, where $\gamma=\left(R_{1} C_{1}\right)^{-1}$. For simplicity we choose $R_{1}=1$ and $C_{1}=1$ and we have the pole $\gamma=-1$, i.e. the transfer function in (1) is normalized.

Applying standard low-pass to band-pass frequency transformation, defined by

$$
s \rightarrow \frac{s^{2}+\omega_{0}^{2}}{B s},
$$

( $\omega_{0}$ is the center frequency and $B$ is the bandwidth of the $\mathrm{BP}$ filter) to the normalized first-order low-pass transfer function as given in (1), we obtain the second-order band-pass transfer function, given by 


$$
T(s)=\frac{K B s}{s^{2}+B s+\omega_{0}^{2}}=\frac{K\left(\omega_{p} / q_{p}\right) s}{s^{2}+\left(\omega_{p} / q_{p}\right) s+\omega_{p}^{2}}
$$

where

$$
\omega_{p}=\omega_{0}, q_{p}=\frac{\omega_{0}}{B}=\frac{1}{B_{n}} .
$$

We present a straightforward realization procedure, with direct element transformation which gives a unique BP filter structure with its component values, as opposite to the standard design procedure in which a designer picks a known BP active filter structure and calculates its elements by comparing the corresponding transfer function parameters with the parameters of the chosen structure [1,2].

To modify low-pass prototype transfer functions and prepare it for a new design method described we apply a frequency transformation [3] on (1):

$$
s=p-\delta \text {, }
$$

where $\delta$ is a real positive constant. We obtain a new transfer function $T_{1}(p)$ with new real pole $p_{p 1}$ in the complex $p$-plane (Fig. 2(b)). The new pole is shifted for amount $\delta$ as shown in the Fig. 2 b). Since the constant $\delta$ can be freely chosen, the pole may lie even in the righthalf $p$-plane. The new LP filter prototype transfer function is

$$
T_{1}(p)=\frac{1}{p+\Gamma}=\frac{K_{1}}{\left(p-p_{p 1}\right)}
$$

where real pole $p_{p 1}=-\Gamma$ is represented by

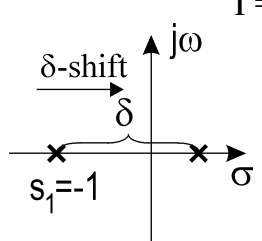

(a)

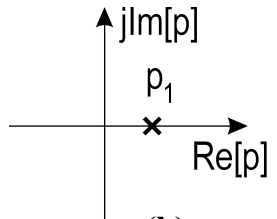

(b)
Fig. 2 Introducing the s-variable transforma-tion. (a) Pole shift for $\delta$. (b) New p-variable.

The transfer function (5) can be realized by first-order circuit, with an operational amplifier shown in Fig. 3.

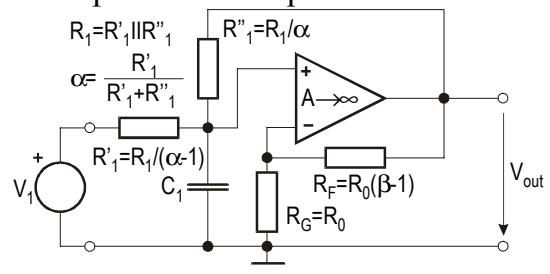

Fig. $31^{\text {st }}$ order active RC low-pass circuit.

Choosing $R_{1}=1$ and $C_{1}=1$, the voltage transfer function $T_{1}(p)$ for this circuit is given by

$$
T_{1}(p)=\frac{V_{\text {out }}}{V_{1}}=\frac{(1-\alpha) \beta}{p+1-\alpha \beta}=\frac{(1-\alpha) \beta}{p+1-\delta}=\frac{\beta-\delta}{p+\Gamma} .
$$

Where the shift $\delta$ equals $\alpha \beta$. Voltage attenuation $\alpha$, $0<\alpha<1$ is realized by splitting input resistor $R_{1}$, and $\beta=1+R_{F} / R_{G} \geq 1$ is the positive amplifier gain. Note that with the circuit shown in Fig. 3, it is possible to realize positive and negative real pole choosing the appropriate values for $\alpha$ and $\beta$.

As shown in [3] a new "lossy"-transformation in the variable $p$ is given by

$$
p \rightarrow \frac{s^{2}+\omega_{0}^{2}}{B_{1} \cdot s}+\delta_{1}
$$

Applied to the transfer function $T_{1}(p)$ it produces the same BP filter transfer function of the form (3).

We also introduce the corresponding impedance transformation which substitutes each resistor of the LP prototype filter by a series resistor and capacitor circuit, and each capacitor by a parallel resistor and capacitor circuit, as shown in Fig. 4, i. e.

$$
\begin{gathered}
R_{1} \rightarrow \frac{1 / R_{a}+s C_{a}}{1 / R_{a} \cdot s C_{a}}=\frac{1}{s C_{a}}+R_{a}, p C_{1} \rightarrow \frac{1}{R_{b}}+s C_{b} . \\
\mathrm{R}_{1}
\end{gathered}
$$

Fig. $4 R C$ impedance transformation as a consequence of the "lossy" LP-BP transform.

Since we choose $R_{1}=1$ and $C_{1}=1$ the substitution given with eq. (8) can be rewritten in the form of the transformation as given with eq. (7), i.e.

$$
p \rightarrow \frac{s^{2}+1 /\left(R_{a} C_{a} R_{b} C_{b}\right)}{s \cdot 1 /\left(R_{a} C_{b}\right)}+\frac{R_{a}}{R_{b}}+\frac{C_{b}}{C_{a}}
$$

Comparing (7) and (9) we have

$$
\omega_{0}^{2}=\frac{1}{R_{a} C_{a} R_{b} C_{b}}, B_{1}=\frac{1}{R_{a} C_{b}}, \delta_{1}=\frac{R_{a}}{R_{b}}+\frac{C_{b}}{C_{a}}
$$

As a result of this procedure we obtain the secondorder band-pass filter shown in Fig. 5.

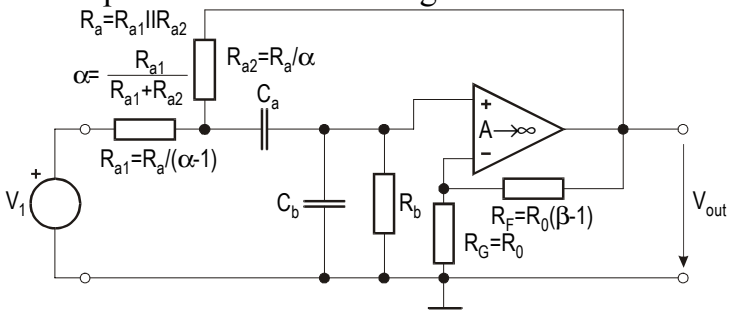

Fig. 5 Second-order band-pass filter circuit (using LP-BP transformation).

The voltage transfer function $T(s)$ for this circuit is obtained by applying the LP-BP transformation (7) on the low-pass prototype transfer function (6), i.e. we have

$$
T(s)=\frac{V_{\text {out }}}{V_{1}}=\frac{(1-\alpha) \beta}{\frac{s^{2}+\omega_{0}^{2}}{B_{1} s}+\delta_{1}-\alpha \beta+1} .
$$


If we introduce the impedance ratios $r$ and $\rho$ (as in [6])

$$
r=R_{a} / R_{b} ; \rho=C_{b} / C_{a}
$$

the expressions in (10) can be rewritten as

$$
\omega_{0}=\frac{1}{R_{b} C_{b}} \sqrt{\frac{\rho}{r}}=\frac{1}{R_{a} C_{a}} \sqrt{\frac{r}{\rho}}, B_{1}=\frac{\omega_{0}}{\sqrt{\rho r}}, \delta_{1}=r+\rho .
$$

Comparison of (11) and (3) gives the pole frequency $\omega_{0}$ and pole $\mathrm{Q} q_{p}$ as

$$
\omega_{p}=\frac{1}{R_{b} C_{b}} \sqrt{\frac{\rho}{r}}, \quad q_{p}=\frac{\omega_{0}}{B_{1} \cdot(\delta+1-\alpha \beta)},
$$

In our design procedure we choose a value of $\beta$

$$
\beta=\frac{\delta}{\alpha}
$$

and from (14) and (3b) we have

$$
B_{1}=B, \delta_{1}=\delta, q_{p}=\frac{\omega_{0}}{B}=\frac{1}{B_{n}} .
$$

The minimum value of constant $\delta$ is limited by the capacitance ratio $\rho=C_{b} / C_{a}$ or the resistor ratio $r=R_{a} / R_{b}$. This ratio can be calculated from (10), and it is

$$
\rho=\frac{C_{b}}{C_{a}}=\frac{\delta}{2} \pm \sqrt{\frac{\delta^{2}}{4}-\frac{\omega_{0}^{2}}{B^{2}}}, r=\frac{R_{a}}{R_{b}}=\frac{\delta}{2} \mp \sqrt{\frac{\delta^{2}}{4}-\frac{\omega_{0}^{2}}{B^{2}}} \text {. }
$$

Since the expression under the square root must be positive, a realizability constraint on the value of the constant $\delta$ is

$$
\delta \geq \delta_{\min }=2 \frac{\omega_{0}}{B}=\frac{2}{B_{n}} .
$$

From (16) it follows

$$
\delta_{\min }=2 q_{p} .
$$

With (18) expressions (17) can be rewritten as

$$
\rho=\frac{\delta}{2} \pm \sqrt{\left(\frac{\delta}{2}\right)^{2}-\left(\frac{\delta_{\min }}{2}\right)^{2}}, r=\frac{\delta}{2} \mp \sqrt{\left(\frac{\delta}{2}\right)^{2}-\left(\frac{\delta_{\min }}{2}\right)^{2}}
$$

or solving for $\delta$ and $\delta_{\min }$ as

$$
\delta_{\min }=2 \sqrt{r \rho}, \delta=r+\rho .
$$

The designer has many degrees of freedom to realize transfer function in (11) by choosing parameters $\alpha, \beta$ and $\delta$. The main criterion is to minimize the sensitivity of the overall transfer function with respect to the component tolerances. The results of numerical examples given in the reference [3] indicate that this could be the case when $\delta=\delta_{\min }$, i.e. for this case:

$$
\frac{C_{b}}{C_{a}}=\frac{R_{a}}{R_{b}}=\frac{\delta_{\min }}{2}
$$

or

$$
\rho=r=\frac{\delta_{\min }}{2} .
$$

In this paper we investigate the design of low sensitivity analytically in order to find more general answer to this problem. In the following text a Schoeffler sensitivity will be a measure which we will minimize.

\section{Schoeffler sensitivity}

The Schoeffler sensitivity is defined as the sum of the squares of sensitivity functions to all passive elements in the network, i.e.

$$
S=\sum_{i=1}^{m}\left(S_{x_{i}}^{|T(j \omega)|}\right)^{2}=\sum_{i=1}^{m}\left(\sum_{j=1}^{n} f_{j}(\omega) \cdot S_{x_{i}}^{\chi_{j}}\right)^{2},
$$

where $x_{i}$ are passive elements $R_{a 1}, R_{a 2}, R_{b}, C_{a}, C_{b}, R_{G}$ and $R_{F} ; f_{j}(\omega)$ are frequency dependent parametersensitivities. Parameter sensitivities are the sensitivities of the transfer function magnitude $|T(j \omega)|$ to the parameters $\chi_{j}$ (i.e. $\delta, \alpha, \beta, B$ and $\omega_{0}$ ) defined as

$$
f_{j}(\omega)=S_{\chi_{j}}^{|T(j \omega)|}=\operatorname{Re}\left[S_{\chi_{j}}^{T(s)}\right]_{s=j \omega}
$$

and presented in Table 1. They depend on the denominator coefficients, of the initial transfer function and some of them on parameter $\delta$. On the other hand parameters $\chi_{j}$ are $\delta, \alpha, \beta, B$ and $\omega_{0}$, are expressed by the passive components (see (10)). We define parameter-tocomponent sensitivities $S_{x_{i}}^{\chi_{j}}$, and present them in Table 2. Both sensitivities, i.e. $f_{j}(\omega)$ and $S_{x_{i}}^{\chi_{j}}$ form Schoeffler sensitivity expression (23).

The band-pass transfer function (11) has the magnitude

$$
|T(j \omega)|=\frac{|N(j \omega)|}{|D(j \omega)|}=\frac{(1-\alpha) \beta}{\sqrt{\left(\frac{\omega_{0}^{2}-\omega^{2}}{B \omega}\right)^{2}+(\delta-\alpha \beta+1)^{2}}}
$$

Our objective is to find a combination of parameters $\delta$, $\alpha, \beta, B$ and $\omega_{0}$, which satisfies the transfer function (25) and in the same time makes the expression (23) minimal.

\begin{tabular}{c|c}
\hline$\chi_{j}$ & $f_{j}(\omega)$ \\
\hline$\omega_{0}$ & $2 \frac{\omega_{0}^{2}}{B^{2}}\left[1-\left(\frac{\omega_{0}}{\omega}\right)^{2}\right] /|D(j \omega)|^{2}$ \\
\hline$B$ & $\frac{\omega_{0}^{2}}{B^{2}}\left[\frac{\omega_{0}}{\omega}-\frac{\omega}{\omega_{0}}\right]^{2} /|D(j \omega)|^{2}$ \\
\hline$\delta$ & $-\delta /|D(j \omega)|^{2}$ \\
\hline$\alpha$ & $\alpha /(\alpha-1)+\delta /|D(j \omega)|^{2}$ \\
\hline$\beta$ & $1+\delta /|D(j \omega)|^{2}$ \\
\hline
\end{tabular}

Table 1 Parameter sensitivities.

Note that if we change one of these parameters, for example $\delta$, the other ones, i.e. $\alpha$ and $\beta$ must be changed in such a way that the overall transfer function $|T(j \omega)|$ 
(and denominator $|D(j \omega)|$ ) given with (25) remain constant [4].

Observing expressions in Table 1, we see that some of them are proportional to the factor $\delta$. Others are inversely proportional to the squared bandwidth $B$ (i.e. directly proportional to the squared pole $\mathrm{Q}$ factor, $q_{p}$ ). Choosing the smallest possible value for $\delta$ reduces the parameter-sensitivity and consequently the overall Schoeffler sensitivity. From (18) it is obvious that we choose $\delta_{\min }$ for the minimum value of $\delta$. The sensitivity will also be smaller for the transfer functions with smaller pole Q factors.

\begin{tabular}{c|c|c|c|c|c}
\hline & \multicolumn{5}{|c}{$S_{x_{i}}^{\chi_{j}}$} \\
\hline$x_{i}$ & $\omega_{0}$ & $B$ & $\delta$ & $\alpha$ & $\beta$ \\
\hline$R_{a 1}$ & $-(1 / 2)(1-\alpha)$ & $-1+\alpha$ & $(r / \delta)(1-\alpha)$ & $1-\alpha$ & \\
\hline$R_{a 2}$ & $-(1 / 2) \alpha$ & $-\alpha$ & $(r / \delta) \alpha$ & $-1+\alpha$ & \\
\hline$R_{b}$ & $-1 / 2$ & & $-r / \delta$ & & \\
\hline$C_{a}$ & $-1 / 2$ & & $-\rho / \delta$ & & \\
\hline$C_{b}$ & $-1 / 2$ & -1 & $\rho / \delta$ & & \\
\hline$R_{F}$ & & & & & $1-1 / \beta$ \\
\hline$R_{G}$ & & & & & $-1+1 / \beta$ \\
\hline
\end{tabular}

Table 2 Parameter-to-component sensitivities.

\section{Design Example}

As an illustration of the proposed BP filter design procedure, we consider the practical example of secondorder band-pass filter with Butterworth transfer function, which has $f_{p}=86 \mathrm{kHz}, q_{p}=1 / \sqrt{2}, K=1$. The design can be carried out by the following step-by-step design procedure:

i) Starting from the second-order BP filter pole $Q$, choose $\delta$ such that (18) is satisfied and calculate impedance scaling factors $r$ and $\rho$ :

If we choose $\delta=\delta_{\text {min }}$ then we have $\rho=r$. With $q_{p}=0.7071$ from (19) we have $\delta=\delta_{\min }=2 q_{p}=1.4142$ and from (22b) $r=\rho=\delta_{\min } / 2=0.7071$ (circuit No.1). If we choose $\delta>\delta_{\text {min }}$ (for circuits No. 2-5) then we calculate $r$ and $\rho$ from (20).

ii) Calculate the new low-pass prototype by shifting the poles by $\delta$ : Applying (4), the new LP prototype function $T_{1}(p)$ pole is: $\Gamma=-0.4142$ As we see $\Gamma$ is negative, i.e., the pole lies in the right-half $p$-plane. However, when applying the "lossy" transformation, the pole will be shifted back into the left-half plane by $\delta$.

iii) Realize the new low-pass prototype circuit components:

To realize negative pole $\Gamma$ we only have to calculate gain $\beta$. If we choose $\alpha=0.5$, then from (15) it follows $\beta=\delta / \alpha=2.83$. iv) Starting from the second-order BP filter pole frequency $\omega_{p}$ and from (14) choose the capacitor $C_{b}$ and calculate the resistor $R_{b}$ :

We choose $C_{b}=500 \mathrm{pF}$ and with $f_{p}=86 \mathrm{kHz}$ it follows $R_{b}=1 /\left(C_{b} \cdot \omega_{p}\right) \cdot \sqrt{\rho / r}=1 /\left(500 \cdot 10^{-12} \cdot 2 \pi \cdot 86 \cdot 10^{3}\right)=3.7 \mathrm{k} \Omega$.

v) Calculate the components of the second-order BP filter:

Using (12) the component values of the $R C$ series and parallel circuits follow $C_{a}=C_{b} / \rho=707 \mathrm{pF} ; R_{a}=r \cdot R_{b}=$ $2.617 \mathrm{k} \Omega$; With $\alpha=0.5$ from step iii) (i.e. $R_{a 1}=R_{a 2}=2 R_{a}$ ) we have $R_{a 1}=R_{a} /(1-\alpha)=5.234 \mathrm{k} \Omega ; R_{a 2}=R_{a} / \alpha=5.234 \mathrm{k} \Omega$. Let $R_{G}=10 \mathrm{k} \Omega$, then $R_{F}=R_{G}(\beta-1)=18 \mathrm{k} \Omega$. Note that the value of $\beta=2.82843$ (i.e. we choose $R_{G}=10 \mathrm{k} \Omega$ and $R_{F}=18.28 \mathrm{k} \Omega$ ) remains the same as in the $\delta$-shifted lowpass prototype, (also the feedback attenuator $\alpha=0.5$ remains the same). A check for the correctness of the resulting filter circuit in the example was performed using PSPICE. Fig. 6 shows the magnitude of the transfer function $\alpha(\omega)=20 \log |T(j \omega)|[\mathrm{dB}]$ of the filter circuit in Fig. 5. Referring to Fig. 5 the resulting filter has the values given in line 1 of Table 3 .

In order to analyze the influence of the shift-constant $\delta$ in "lossy" transformation on the sensitivities to component tolerances, and to find an optimal value of $\delta$, five different realizations corresponding to three values of $\delta$ are analyzed.

\begin{tabular}{c|c|c|c|c|c|c|c|c|c}
\hline No. & $R_{a}$ & $R_{b}$ & $r$ & $C_{a}$ & $C_{b}$ & $\rho$ & $\beta$ & $\delta$ & $\delta_{\min }$ \\
\hline 1$)$ & 2.62 & 3.7 & 0.71 & 707 & 500 & 0.71 & 2.83 & 1.41 & 1.41 \\
\hline 2$)$ & 2.62 & 8.94 & 0.29 & 293 & 500 & 1.71 & 4.0 & 2.0 & 1.41 \\
\hline 3$)$ & 2.62 & 1.53 & 1.71 & 1707 & 500 & 0.29 & 4.0 & 2.0 & 1.41 \\
\hline 4$)$ & 2.62 & 14.8 & 0.18 & 177 & 500 & 2.82 & 6.0 & 3.0 & 1.41 \\
\hline 5$)$ & 2.62 & 0.93 & 2.82 & 2823 & 500 & 0.18 & 6.0 & 3.0 & 1.41 \\
\hline
\end{tabular}

Table 3 Component values of second-order filter as in Fig. 5 (resistors in $\mathrm{k} \Omega$, capacitors in pF).

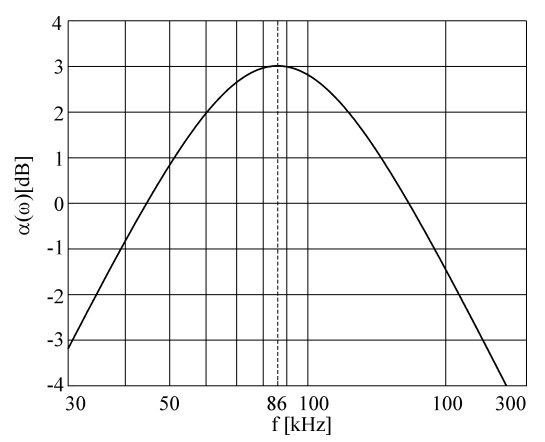

Fig. 6 Magnitude of second-order band-pass filter as in Fig. 5 (line 1 of Table 3).

Parameter sensitivities as defined in Table 1, for five different realizations in Table 3, are presented in Fig. 7. 

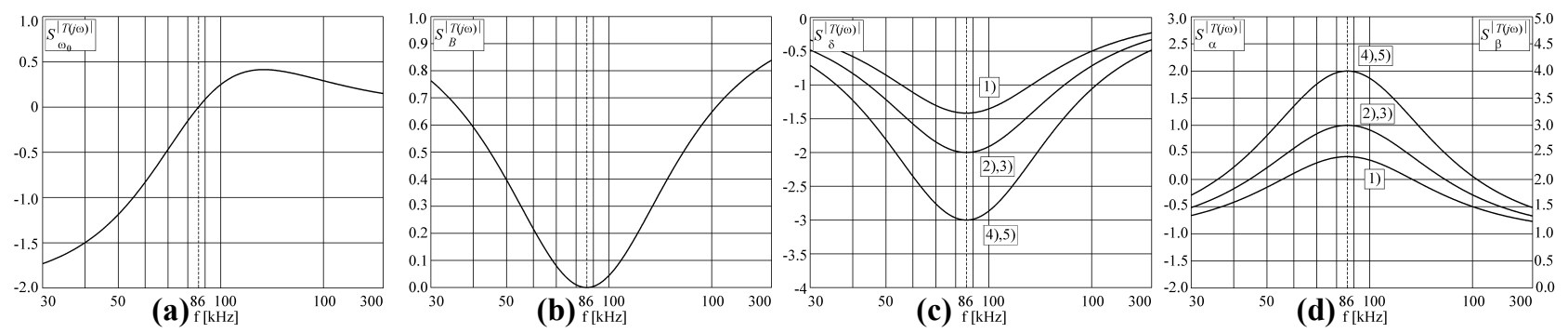

Fig. 7 Parameter sensitivities for filters in Table 3. Transfer function magnitude sensitivity to (a) $\omega_{0}$. (b) B. (c) $\delta$. (d) $\alpha$ and $\beta$.

It can be seen that sensitivities to $\omega_{0}$ and $B$ are identical for all cases. The sensitivity to parameter $\delta$ is minimal when $\delta=\delta_{\min }$.

An overall sensitivity analysis was performed. The standard deviation (which is related to the Shoeffler sensitivities) of the variation of the logarithmic gain $\Delta \alpha=8.68588 \Delta\left|T_{B P}(\omega)\right| /\left|T_{B P}(\omega)\right|$, with respect to zero mean and $1 \%$ standard deviation of the components, was calculated and shown in Fig. 8. Although having the same value of $\delta$, note that sensitivities for pairs of circuits No. 2), 3) and 4), 5) slightly differ, because the input resistor $R_{a}$ is split into $R_{a 1}$ and $R_{a 2}$ to realize the voltage attenuator $\alpha$. The difference in sensitivity can be approved using eq. (23).

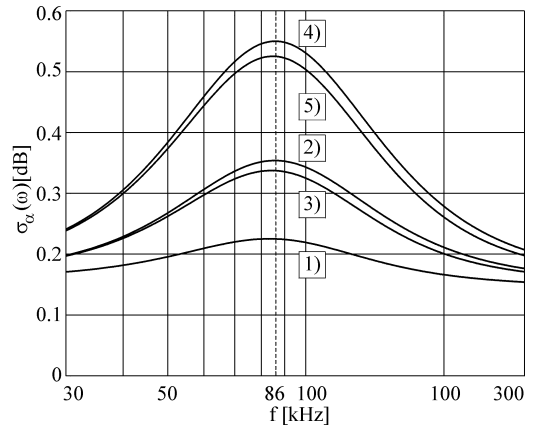

Fig. 8 Schoeffler sensitivities for realizations in Table 3.

As can be seen the best results are obtained for the value of the shift parameter $\delta=\delta_{\min }$, i.e. circuit No. 1). Monte Carlo runs, carried out for the same examples, confirmed this result.

\section{Conclusions}

A procedure for the design of low-power second-order allpole band-pass active- $R C$ filters is presented. The design is based on a low-pass to band-pass transformation, which is applied to a first-order low-pass filter prototype. The amplifier of the second-order band- pass filter provides a low output impedance and supplies positive feedback to the passive $R C$-network. It is shown that a "lossy" LP-BP transformation transforms the resistors of the low-pass prototype circuit into series resistor-capacitor combinations, and capacitors into parallel resistor-capacitor combinations, resulting in a single-amplifier second-order band-pass filter circuit. Detailed closed-form design equations for this circuit are given. In summary, for the second-order allpole Class-4 $[2,5]$ band-pass filter, ideal impedance scaling with $\rho=r$ provides circuits with minimum sensitivity to the component tolerances of the circuit.

\section{References}

[1] G. S. Moschytz, Linear Integrated Networks: Design. New York (Bell Laboratories Series): Van Nostrad Reinhold Co., 1975.

[2] G. S. Moschytz and P. Horn, Active Filter Design Handbook. Chichester, U.K.: Wiley 1981.

[3] D. Jurišić, N. Mijat, G. S. Moschytz, Design of Fourth-Order Band-Pass Active-RC Filters Using a "Lossy" Low-pass to Band-pass Transformation, Accepted for presentation on European Conference on Circuit Theory and Design, ECCTD, August 28 31, 2001 Espoo, Finland.

[4] N. Mijat, Low Sensitivity Structures in Realization of Active Filters. Ph. D. Dissertation, University of Zagreb, Zagreb, Croatia, October, 1984.

[5] R. P. Sallen and E. L. Key, "A practical Method of Designing $R C$ Active Filters," IRE Transactions on Circuit Theory, vol. CT-2, pp. 78-85, 1955.

[6] G. S. Moschytz, "Low-Sensitivity, Low-Power, Active-RC Allpole Filters Using Impedance Tapering," IEEE Trans. on Circuits and Systems, vol. CAS-46, No. 8, pp. 1009-1026, Aug. 1999. 